\title{
Osmoregulants Involved in Osmotic Adjustment for Differential Drought Tolerance in Different Bentgrass Genotypes
}

\author{
Nanqing Liu \\ College of Agro-Grassland Science, Nanjing Agricultural University, Nanjing 210095, P.R. China; \\ Department of Landscape Architecture, Jiangsu Polytechnic College of Agriculture and Forestry, \\ Jurong 212400, P.R. China; and Department of Plant Biology and Pathology, Rutgers University, \\ New Brunswick, NJ 08901 \\ Yixin Shen ${ }^{1}$ \\ College of Agro-Grassland Science, Nanjing Agricultural University, Nanjing 210095, P.R. China; \\ and Department of Landscape Architecture, Jiangsu Polytechnic College of Agriculture and Forestry, \\ Jurong 212400, P.R. China \\ Bingru Huang ${ }^{1}$ \\ Department of Plant Biology and Pathology, Rutgers University, New Brunswick, NJ 08901 \\ Additional Index words. Agrostis stolonifera, osmotic potential, solutes, water stress

\begin{abstract}
Compatible solute accumulation regulating osmotic adjustment (OA) is associated with drought tolerance. The objectives of this study were to examine genetic variations in OA among a diverse group of bentgrass (Agrostis sp.) genotypes or lines with differential drought tolerance, and determine major types of organic osmoregulants contributing to $\mathrm{OA}$ and accounting for the genetic variations in drought tolerance. A wild type cultivar of creeping bentgrass [Agrostis stolonifera (Penncross)], a transgenic line of creeping bentgrass (SAGIPT41), and four hybrid bentgrass lines [Agrostis capillaris $\times$ Agrostis stolonifera $($ ColxCr14, ColxCr190, ColxCr481, and ColxCr679)] were exposed to drought stress by withholding irrigation for 17 days in growth chambers. Among genotypes, ColxCr14, ColxCr190, and SAGIPT41 showed superior drought tolerance, as manifested by higher turf quality (TQ) and leaf relative water content (RWC), as well as $O A$ than 'Penncross', ColxCr679, and ColxCr481 under drought stress. SAGIPT41 leaves accumulated greater content of soluble sugars (glucose, sucrose, and fructose), proline, glycine betaine (GB), and spermine; ColxCr190 had higher content of soluble sugars and spermidine; and ColxCr14 accumulated more soluble sugars and GB, compared with the three drought-sensitive genotypes. Soluble sugars were predominant contributors to OA, followed by GB and proline, with all three forms of polyamine (PA) as minor contributors in bentgrass genotypes. The osmolytes highly correlated to $\mathrm{OA}$ and superior drought tolerance could be used as biomarkers to select for drought-tolerant germplasm of bentgrass and other cool-season turfgrass species.
\end{abstract}

Drought stress damage in plants is characterized of leaf dehydration, and therefore, increasing the capacity of water retention in leaves or improving leaf tolerance to dehydration to maintain cell turgor is critically important for plant survival of drought stress. Dehydration tolerance has been associated with the accumulation of compatible solutes in plant cells, which accounts for OA (Chaves et al., 2003). Osmotically active solutes or osmoregulants for OA regulate either movement of water into or reduced water efflux from cells, and thus help to maintain cellular turgor, enabling tissues to sustain growth and metabolic and physiological functions under drought stress (Bohnert and Jensen, 1996; Hare et al., 1998). In addition to the involvement in OA, the accumulation of compatible solutes also play roles in stabilizing proteins and membranes against

Received for publication 7 July 2015. Accepted for publication 7 Oct. 2015. Thanks go to Jiang Su Education Commission for financial support for Nanqing Liu to conduct the collaborative research project at Rutgers University. Authors also wish to thank Patrick Burgess for assistance in plant material preparation and technical support.

'Corresponding author. E-mail: yxshen@njau.edu.cn or huang@aesop.rutgers. edu. desiccation injury, as well as protection against oxidative damage (Arakawa et al., 1991; Hoekstra et al., 2001; Rhodes and Hanson, 1993). The types of osmoregulants reported in OA are diverse, and typically include low molecular weight compounds such as amino acids (e.g., proline), ammonium compounds (e.g., GB and PA), sugars (e.g., fructose and sucrose), and organic acids (e.g., malate), as well as inorganic ions (e.g., potassium and calcium) (Chaves et al., 2003).

Improvement in drought tolerance has been positively correlated with OA in leaves of many plant species, including perennial grasses used as turfgrasses (DaCosta and Huang, 2006; Qian and Fry, 1997; White et al., 1992). Qian and Fry (1997) reported interspecific variations in OA among three warm-season and one cool-season turfgrass species, with OA ranking as 'Prairie' buffalograss (Buchloe dactyloides) = 'Meyer' zoysiagrass (Zoysia japonica) > 'Midlawn' hybrid bermudagrass (Cynodon dactylon $\times$ Cynodon transvaalensis) $>$ 'Mustang' tall fescue (Festuca arundinacea). Interspecific variations in OA associated with drought tolerance has also been reported among cool-season turfgrass species, such as greater OA in velvet bentgrass (Agrostis canina) leaves than creeping bentgrass (DaCosta and Huang, 2006). White et al. 

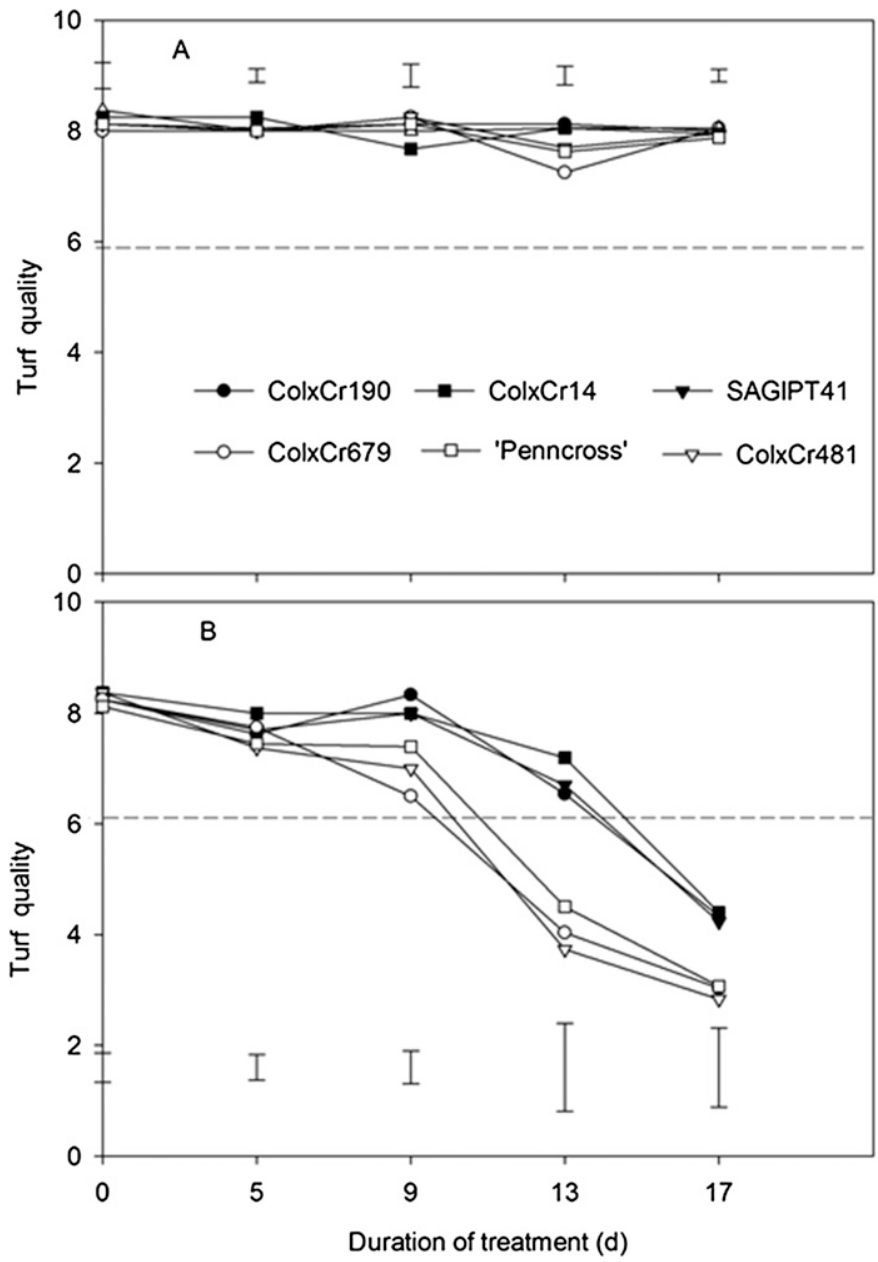

Fig. 1. Turf quality of six genotypes of bentgrass under (A) well-watered conditions and (B) drought stress rated on a scale of $1-9[1=$ completely desiccated brown turf canopy; $9=$ healthy plants with dark green, turgid leaf blades, and a dense turf canopy (Turgeon, 2008)]. Vertical bars on the top are least significant difference values $(P=0.05)$ for comparisons at a given day of treatment.

(1992) found genotypic variations in OA within a species, such as for tall fescue leaves, which was positively associated with tiller survival of drought stress. Despite of knowledge in the diverse genetic variability of $\mathrm{OA}$ among turfgrass grass species and within genotypes of the same species in the degree of OA, the specific types of osmoregulants accounting for the genetic variations in $\mathrm{OA}$ and associated with differential drought tolerance are not well documented. Understanding major osmoregulants contributing to OA may identify potential biomarkers or candidate genes to select for germplasm with high capacity of OA and improved drought tolerance.

Bentgrass species widely used turfgrass species on golf courses, including creeping bentgrass and colonial bentgrass, vary genetically in drought tolerance (DaCosta and Huang, 2006). Some hybrid lines of colonial bentgrass $\times$ creeping bentgrass exhibited superior drought tolerance relative to traditional creeping bentgrass genotypes, such as 'Penncross' (Merewitz et al., 2012). Transgenic creeping bentgrass over-expressing a gene controlling cytokinin synthesis was found to exhibit improvement in OA and drought tolerance compared with the wild type 'Penncross' (Merewitz et al., 2011). However, major

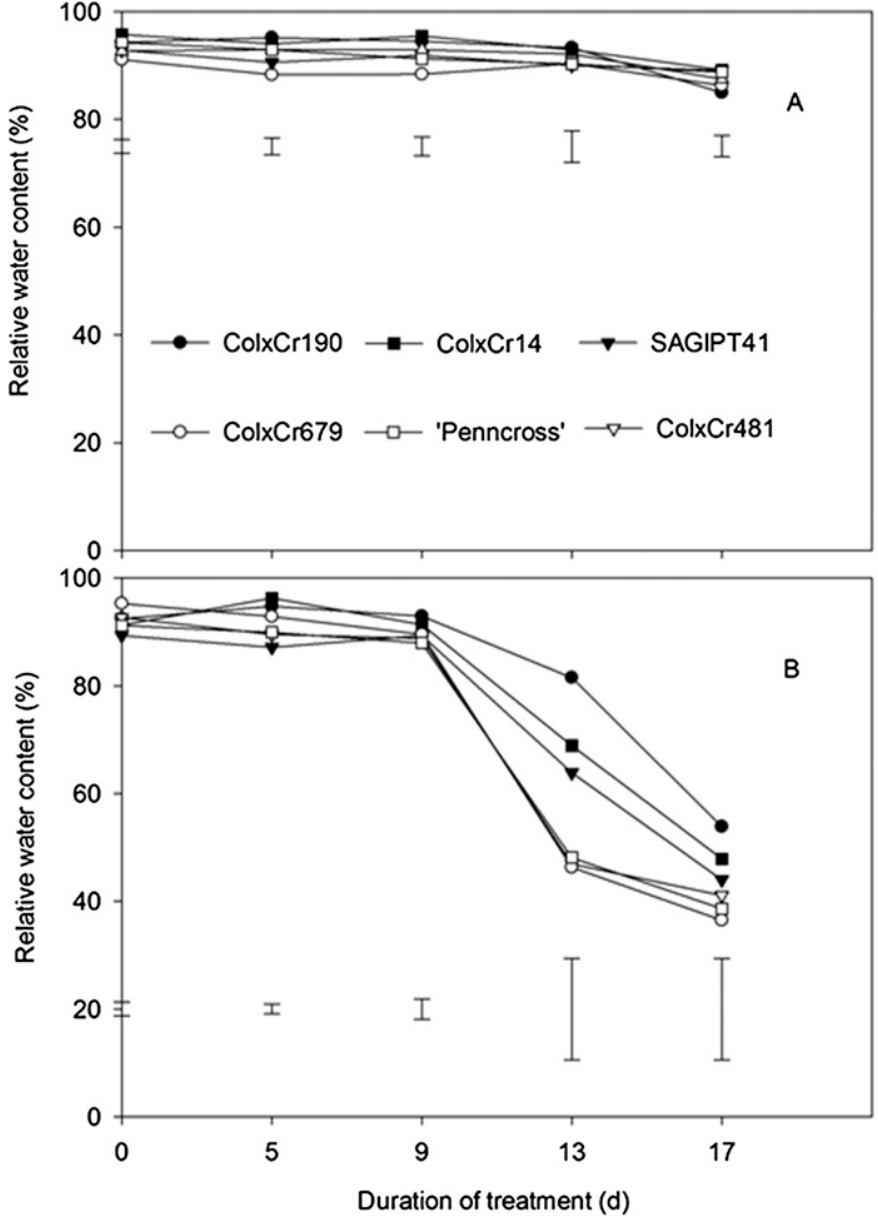

Fig. 2. Leaf relative water content of six genotypes of bentgrass under (A) wellwatered conditions and $(\mathbf{B})$ drought stress. Vertical bars at the bottom are least significant difference values $(P=0.05)$ for cultivar comparisons at a given day of treatment.

osmoregulants may account for the genetic variations in $\mathrm{OA}$ and associated drought tolerance among the diverse germplasm of bentgrass is yet to be determined. The objectives of this study were to examine genetic variations in OA among a diverse group of bentgrass genotypes or lines with differential drought tolerance, and determine major types of organic osmoregulants contributing to $\mathrm{OA}$ and accounting for the genetic variations in drought tolerance. Six genotypes of bentgrass that exhibited diverse genetic variations in drought tolerance levels were examined in the study, including a wild type cultivar of creeping bentgrass (Penncross), a transgenic line of creeping bentgrass overexpressing a gene (ipt encoding isopentenyl transferase) controlling cytokinins synthesis (SAGIPT41), and four hybrid lines colonial bentgrass $\times$ creeping bentgrass (ColxCr14, ColxCr190, ColxCr481, and ColxCr679). SAGIPT41 plants have been previously reported to exhibit superior drought tolerance to 'Penncross' (Merewitz et al., 2011), but their relative drought tolerance to the hybrid genotypes are unknown. Through using genotypes with a wide range of drought tolerance levels, specific osmoregulants associated with improved OA could be identified.

\section{Materials and Methods}

Plant materials and growth conditions. Sods of SAGIPT41, four hybrid lines of colonial bentgrass $\times$ creeping 

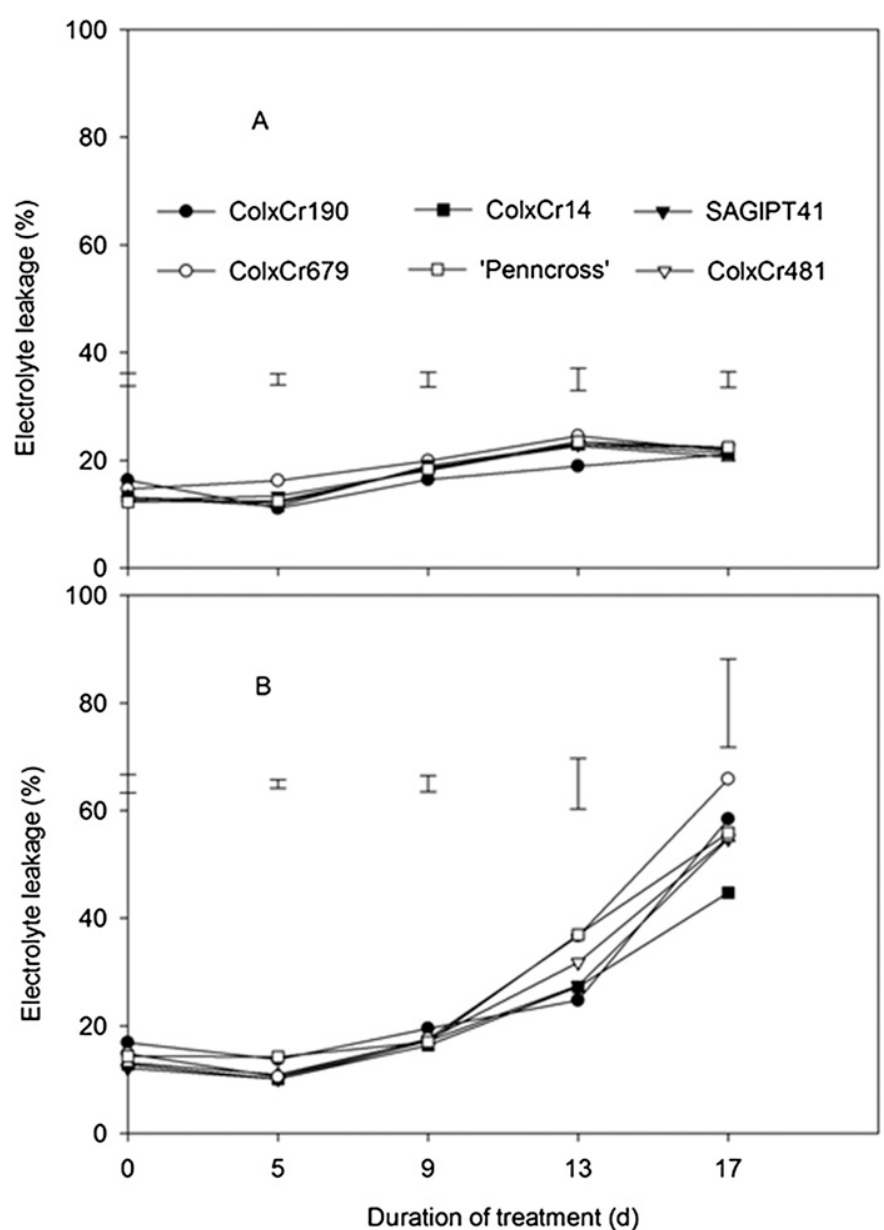

Fig. 3. Leaf electrolyte leakage of six genotypes of bentgrass under (A) wellwatered conditions and (B) drought stress. Vertical bars at the bottom are least significant difference values $(P=0.05)$ for cultivar comparisons at a given day of treatment.

bentgrass (ColxCr14, ColxCr190, ColxCr481, and ColxCr679), and 'Penncross' were transplanted from field plots into polyvinyl containers (40-cm wide, $80-\mathrm{cm}$ long, and $40-\mathrm{cm}$ deep) in a greenhouse at Rutgers University (New Brunswick, NJ) on 3 Mar. 2013. SAGIPT41 and the four hybrid lines were selected at Rutgers University and 'Penncross' was developed by The Pennsylvania State University. Each container was separated into 12 compartments with polyethylene foam plates, which was filled with fritted clay (Turface Green Grade; Profile Products, Buffalo Grove, IL). Two sets of plants of each of six genotypes were randomly planted in the 12 compartments within each container. Holes $(\approx 1.5 \mathrm{~mm}$ diameter) were drilled on the polyethylene divider, so water could move freely across compartments within each container while roots of different genotypes were prevented to be tangled together. This setup enabled all genotypes exposed to equivalent level of soil water content during drought stress as water could move or diffuse across different compartments. Plants were maintained in a greenhouse under 10 to $12 \mathrm{~h}$ natural light conditions and average temperatures of $21 / 13^{\circ} \mathrm{C}$ (day/night) for 3 months during Mar. to May 2013 and then moved to a walk-in growth chamber (Environmental Growth Chamber, Chargrin Falls, $\mathrm{OH}$ ) where treatments were imposed on 6 May 2013. The growth chamber was maintained at

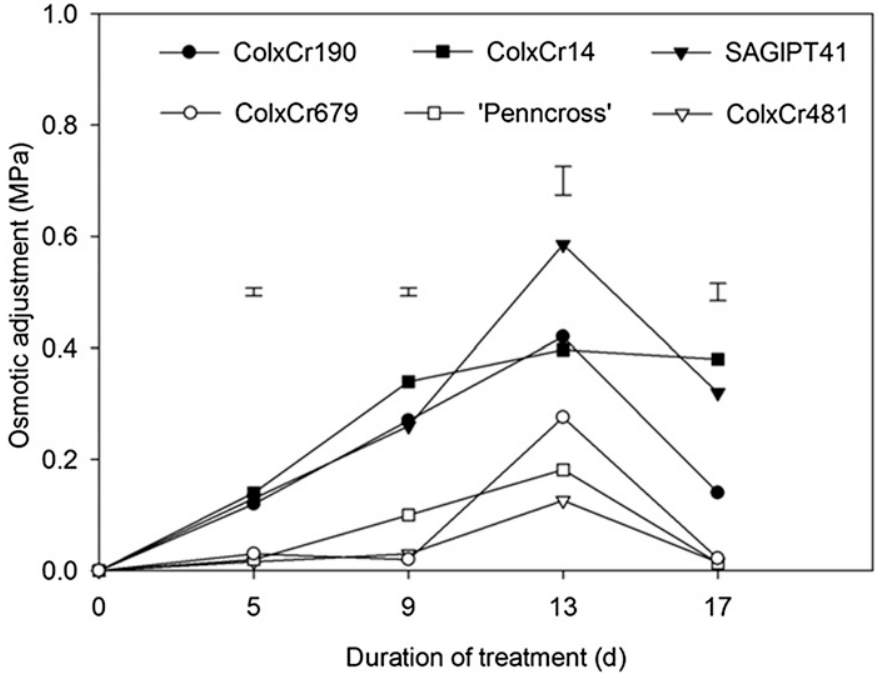

Fig. 4. Osmotic adjustment of six genotypes of bentgrass at $13 \mathrm{~d}$ of drought stress. Vertical bars at the bottom are least significant difference values $(P=$ 0.05 ) for cultivar comparisons at a given day of treatment.

Table 1. Correlation of osmotic adjustment (OA) with turf quality (TQ), relative water content (RWC), and electrolyte leakage (EL) for all bentgrass genotypes at $13 \mathrm{~d}$ of drought stress.

\begin{tabular}{lllc}
\hline & TQ & RWC & EL \\
\hline OA & $0.73^{*}$ & $0.60^{*}$ & $-0.55^{*}$ \\
TQ & 1 & $0.80^{*}$ & $-0.63^{*}$ \\
RWC & & 1 & $-0.78^{*}$ \\
\hline
\end{tabular}

*Correlation significant at $P<0.01$.

$20 / 15{ }^{\circ} \mathrm{C}$ (day/night), $70 \%$ relative humidity, 12 -h photoperiod, and photosynthetically active radiation of $650 \mu \mathrm{mol} \cdot \mathrm{m}^{-2} \cdot \mathrm{s}^{-1}$ at canopy height. Plants in the containers were watered three times per week to maintain soil moisture at field capacity until drainage occurred from the bottom of the containers, and fertilized weekly with half-strength Hoagland's solution (Hoagland and Arnon, 1950) before exposure to drought. Grasses were cut at $6 \mathrm{~cm}$ height every $2 \mathrm{~d}$ with scissors, with clippings removed.

TREATMENTS AND EXPERIMENTAL DESIGN. The six bentgrass genotypes were exposed to two soil water treatments: 1) wellwatered control: plants were watered every other day to soil reaching field capacity (soil volumetric water content at $28 \%$ ); and 2) drought stress: irrigation was withheld for $17 \mathrm{~d}$ until soil volumetric water content declined to $10 \%$, which was monitored using the time domain reflectometer (Trase; Soil Moisture Equipment, Santa Cruz, CA). Each water treatment was replicated in four containers. Each container included two sets (or subsamples) of plants for each genotype. The genotype and water treatments were arranged as a split-plot design with water treatments as main plots and genotype as subplots. Containers with well-watered or drought-stressed plants were rotated in the growth chamber to minimize variability due to the environment.

Statistical significance of data were tested using the analysis of variance procedure (SAS version 9.0; SAS Institute, Cary, NC). Differences between treatment means and genotype were separated by Fisher's protected least significance difference test at the $0.05 P$ level. 

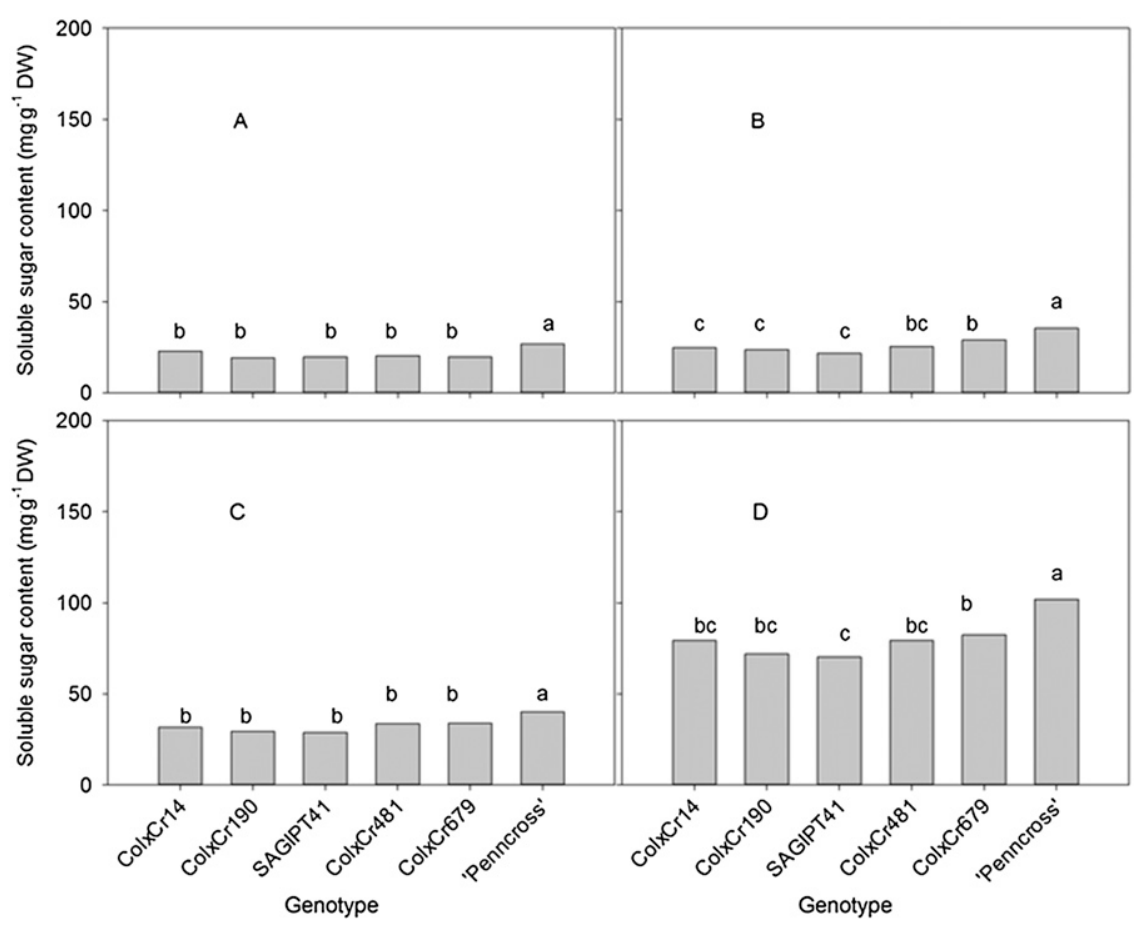

Fig. 5. Content of (A) glucose, (B) sucrose, (C) fructose, and (D) total soluble sugars for six genotypes of bentgrass under well-watered conditions. Columns marked with different letters indicate significant differences among genotypes for each parameter based on least significant difference test $(P=0.05)$.

Growth AND Physiological EVALUATION OF GENETIC VARIATION IN DROUGHT TOLERANCE. Several commonly used parameters were used to evaluate genetic variations in growth and physiological response to drought stress, including visual evaluation of TQ, leaf RWC, electrolyte leakage (EL), and leaf photochemical efficiency. Measurements were taken during the month of June and July.

TQ was visually rated on a scale of 1 to 9 with a rating of 1 being a completely desiccated brown turf canopy and a rating of 9 representing healthy plants with dark green, turgid leaf blades, and a dense turf canopy (Turgeon, 2008). A rating of 6 was considered the minimal acceptable TQ level.

RWC was calculated using the formula: $100 \times[(\mathrm{FW}-\mathrm{DW}) /$ (TW - DW)] (Barrs and Weatherley, 1962), where FW is the leaf fresh weight, TW is the leaf turgid weight, and DW is the leaf dry weight after oven-drying leaf samples for $72 \mathrm{~h}$ at $80^{\circ} \mathrm{C}$. TW was determined as weight of fully turgid leaves after soaking leaves in distilled water in the refrigerator for $24 \mathrm{~h}$.

Cellular membrane stability was evaluated based on EL (Blum and Ebercon, 1981). Leaves $(\approx 0.15 \mathrm{~g}$ ) were cut to $0.5-\mathrm{cm}$ long segments, soaked in $30 \mathrm{~mL}$ of deionized water, and placed on a conical shaker. Following 12-h incubation, initial level of EL $\left(\mathrm{C}_{\mathrm{i}}\right)$ was measured using a conductance meter (model 132; Yellow Springs Instrument Co., Yellow Springs, OH). Leaf samples were then killed at $120{ }^{\circ} \mathrm{C}$ for $15 \mathrm{~min}$ in an autoclave, incubated in deionized water on the conical shaker for $12 \mathrm{~h}$, and final level of conductance of the incubation solution $\left(\mathrm{C}_{\max }\right)$ was measured. Leaf EL was calculated as $\left(\mathrm{C}_{\mathrm{i}} / \mathrm{C}_{\max }\right) \times 100$ (Blum and Ebercon, 1981).

OA ANALYSIS. OA $\left(\Psi_{\pi 100}\right)$ was determined according to the rehydration method, in which $\Psi_{\pi 100}$ of leaves was determined after soaking in water for full rehydration (Blum, 1989; Blum and Sullivan, 1986). Turgid leaf samples were frozen in liquid nitrogen and subsequently stored at $-20^{\circ} \mathrm{C}$ until analysis of leaf $\Psi_{\text {s }}$ at full turgor $\left(\Psi_{\pi 100}\right)$. Frozen leaf samples were thawed and cell sap was pressed from leaves using a laboratory press (Fred S. Carver, Wabash, IN), which was subsequently analyzed for osmolality [C (millimoles per kilogram)] using a vapor pressure osmometer (Vapro model 5520; Wescor, Logan, UT). Osmolality of cell sap was converted from millimoles per kilogram to $\Psi_{\pi}$ [megapascals (MPa)] using the formula: $\mathrm{MPa}=-\mathrm{C} \times 2.58 \times 10^{-3}$. OA was determined as the difference in $\Psi_{\pi 100}$ between well-watered and drought-stressed plants.

Quantification of Solute CONTENT. Approximately 1-2 g fresh leaves from each replicate for each treatment were sampled at $0 \mathrm{~d}$ (wellwatered conditions) and $13 \mathrm{~d}$ of drought stress. Samples were frozen in liquid nitrogen for the analysis of content of major osmoregulants, including soluble sugars, proline, PAs [putrescine (Put), spermidine (Spd), and spermine (Spm)], and GB.

Soluble sugars were determined using the phenol-sulfuric acid method (Buysse and Merckx, 1993). Soluble sugars were extracted from leaves $(\approx 1.0 \mathrm{~g} \mathrm{FW})$. An aliquant of $1-\mathrm{mL}$ leaf extractant was added to $1 \mathrm{~mL}$ phenol solution and mixed well, in which $5 \mathrm{~mL}$ concentrated sulfuric acid (95\%) was added. The reaction solution was incubated in water bath at $30{ }^{\circ} \mathrm{C}$ for $30 \mathrm{~min}$ and then was cool down for $15 \mathrm{~min}$. The absorbance of the reaction solution was read with a spectrophotometer (Spectronic Instruments, New York, NY) at $490 \mathrm{~nm}$. A calibration curve with D-glucose was done as a standard.

Proline content was determined using the ninhydrin method (Magné and Larher, 1992). Fresh leaf samples ( $\approx 300 \mathrm{mg}$ ) were ground in liquid nitrogen and then a $5 \mathrm{~mL}$ sulfosalicylic acid was added into the ground powders; $2 \mathrm{~mL}$ acetic acid and $2 \mathrm{~mL}$ ninhydrin were added to the extractant in test tubes. The test tubes with extractants were placed in a water bath with boiling water for $45 \mathrm{~min}$ and then cooled. The final reaction was completed by adding $4 \mathrm{~mL}$ toluene in the extractants. The absorbance of the resulting organic layer was measured with a spectrophotometer at $520 \mathrm{~nm}$. A calibration curve was made with L-Proline as a standard.

The content of PAs (Put, Spd, and Spm) was determined according to the method of Liu et al. (2002). Dry leaf tissue power $(0.1 \mathrm{~g})$ was extracted in $2 \mathrm{~mL}$ precooled perchloric acid, ice-bath for $60 \mathrm{~min}$ and centrifuged at $15,000 \mathrm{~g}_{\mathrm{n}}$ for $30 \mathrm{~min}$. The supernatant was transferred into a centrifugal tube and stored at $-20{ }^{\circ} \mathrm{C}$. PAs contained in the supernatant were subjected to a benzoylation reaction in the alkaline medium. Benzoyl PA derivatives were extracted by diethyl ether. Ether fraction was evaporated to dryness and dissolved in methanol. Highperformance liquid chromatography (HPLC) was performed on 

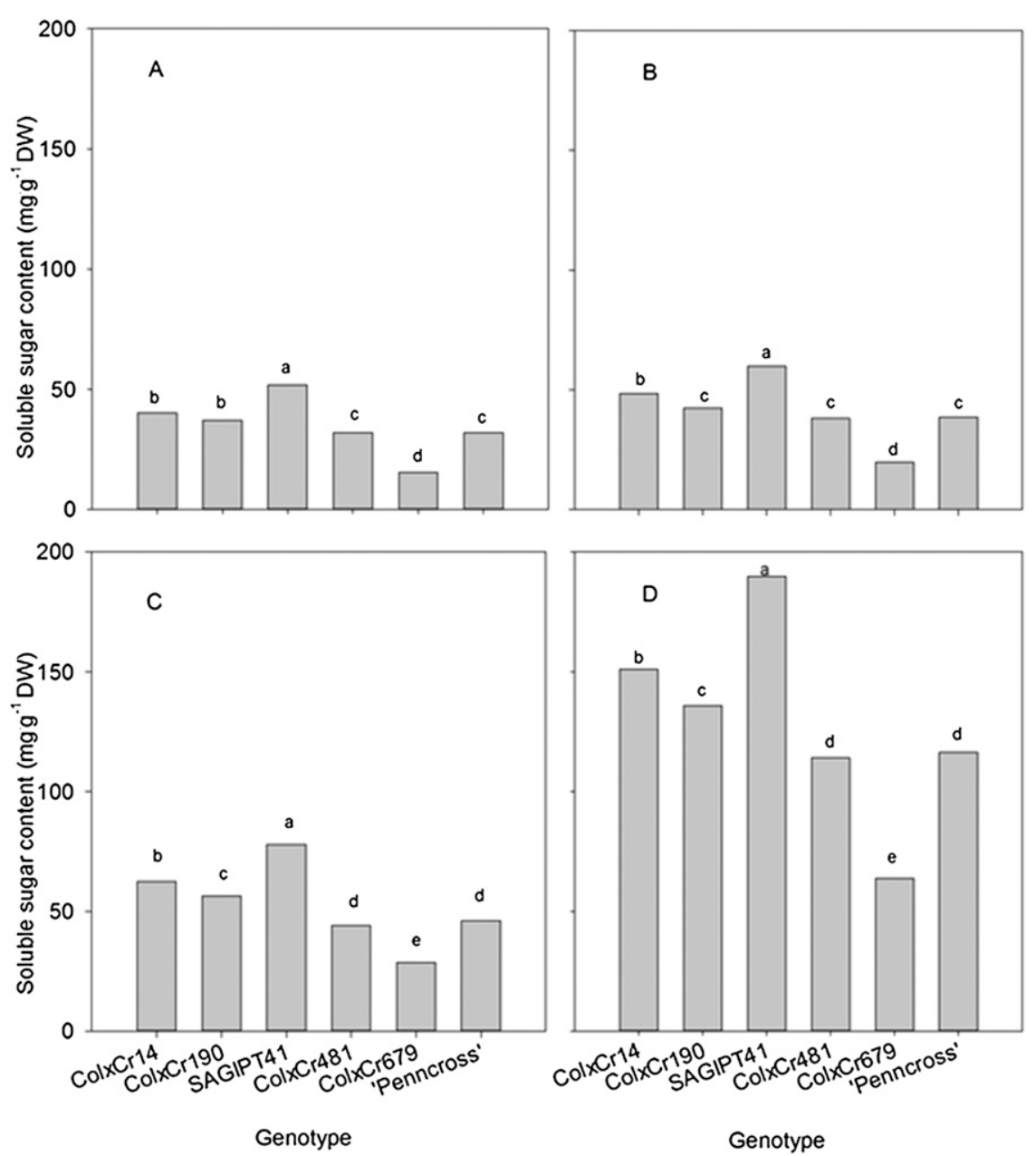

Fig. 6. Content of (A) glucose, (B) sucrose, (C) fructose, and (D) total soluble sugars for six genotypes of bentgrass at $13 \mathrm{~d}$ of drought stress. Columns marked with different letters indicate significant differences among genotypes for each parameter based on least significant difference test $(P=0.05)$.

the liquid chromatograph (Thermo Scientific, Waltham, MA) using a $5-\mu \mathrm{m}, 250 \times 4.6-\mathrm{mm}$ column [Diamonsil $\mathrm{C}^{18}(2)$; Alltech Associates, Deerfield, IL]. A $10 \mu \mathrm{L}$ of methanol solution of benzoyl PAs was injected into an autosampler (Surveyor, Thermo Scientific) every 35 min. Samples were eluted from the column with $70 \%$ methanol and with a temperature maintained at $30{ }^{\circ} \mathrm{C}$, and the flow rate was $0.7 \mathrm{~mL} \cdot \mathrm{min}^{-1}$. PA peaks were detected with an ultraviolet detector at $230 \mathrm{~nm}$. The applied standards were Put, Spd, and Spm.

GB was determined using the method described by Wang et al. (2010). Dry leaf tissue power $(0.1 \mathrm{~g})$ was extracted in 12.5 $\mathrm{mL}$ water, shaked for $30 \mathrm{~min}$, and then centrifuged at $2270 \mathrm{~g}_{\mathrm{n}}$ for $5 \mathrm{~min}$. The supernatant was filtered and filtrate was transferred to solid-phase cartridges $[150 \mathrm{mg} / 6 \mathrm{~mL}$ (Poly-Sery MCX; CNW Technologies, Dusseldorf, Germany)]. Then extraction cartridges were rinsed by methanol/water $(85 / 15 \mathrm{v} / \mathrm{v})$ and methanol. Elution was finished by rinsing with mixture of ammonia water/methanol $(5: 95 \mathrm{v} / \mathrm{v})$ twice. Eluent was condensed dry and diluted with acetonitrile/water $(50 \% \mathrm{v} / \mathrm{v})$ followed by passing through a $0.45-\mu \mathrm{m}$ membrane (Merck Millipore, Darmstadt, Germany) for further analysis in HPLC. Solution of acetonitrile/water $(50 \% \mathrm{v} / \mathrm{v})$ was the mobile phase for HPLC analyses. GB was analyzed and quantified by HPLC using an Atlantis HILIC Silica column $(4.6 \times 150 \mathrm{~mm}$ filled with $5 \mu \mathrm{m}$ particle diameter; Waters Corp., Milford, MA). The peak areas were integrated and compared with standard curve constructed with standard of GB.

The contribution of each solute to OA, calculated as osmolarity [C (millimoles per kilogram)], was expressed as a percentage of $\pi 100$ measured from the same sample. $\pi 100=-0.1013 R T i C$ ( $R$ was gas constant, the value was $0.08314 ; T$ was degree Kelvin, $T=273+$ room temperature; $i C$ was the value that showed on the $\Psi_{\mathrm{s}}$ meter).

\section{Results}

GENOTYPIC VARIATIONS IN PHYSIOLOGICAL RESPONSES TO DROUGHT STRESS. TQ was visually rate to evaluate overall turf performance. TQ maintained at 7.2 or more throughout the treatment period in all genotypes under wellwatered conditions (Fig. 1A). Under drought stress, TQ exhibited a steady decline, and the rate of decline varied among genotypes (Fig. 1B). By $13 \mathrm{~d}$ of drought treatment, TQ of ColxCr481, ColxCr679, and 'Penncross' declined to below the minimum acceptable level (6.0) whereas ColxCr14, SAGIPT41, and ColxCr190 maintained TQ above the minimum acceptable level. During the 17-d drought period, ColxCr14, SAGIPT41, and ColxCr190 had higher TQ than that of ColxCr481, ColxCr679, and 'Penncross'.

Leaf RWC was determined to evaluate leaf hydration status. All well-watered plants maintained RWC above 90\% throughout the treatment period (Fig. 2A). At 13 and $17 \mathrm{~d}$ of drought stress, significant decreases in RWC were observed in all genotypes. During prolonged periods of drought (13 and $17 \mathrm{~d}$ ), ColxCr14, ColxCr190, and SAGIPT41 showed significantly higher RWC than ColxCr679, 'Penncross', and ColxCr481 (Fig. 2B).

Leaf EL was measured to estimate cell membrane stability. All well-watered plants maintained low EL (below 24\%) throughout the treatment period (Fig. 3A). Leaf EL increased with drought stress in all genotypes, but genotypes did not exhibit significant differences in EL during most of the drought period (Fig. 3B).

GENOTYPIC VARIATIONS IN OSMOTIC ADJUSTMENT AND CORRElation to PHYSIOLOGICAL TRAITS. OA level in leaves increased during drought stress in all genotypes (Fig. 4). The OA was significantly greater in ColxCr14, ColxCr190, and SAGIPT41 than ColxCr679, 'Penncross', and ColxCr481 during the entire drought period (Fig. 4).

Correlation analysis between $\mathrm{OA}$ and other physiological parameters was conducted to determine the relationship of OA to TQ, RWC, and EL. Leaf OA was positively correlated to TQ 

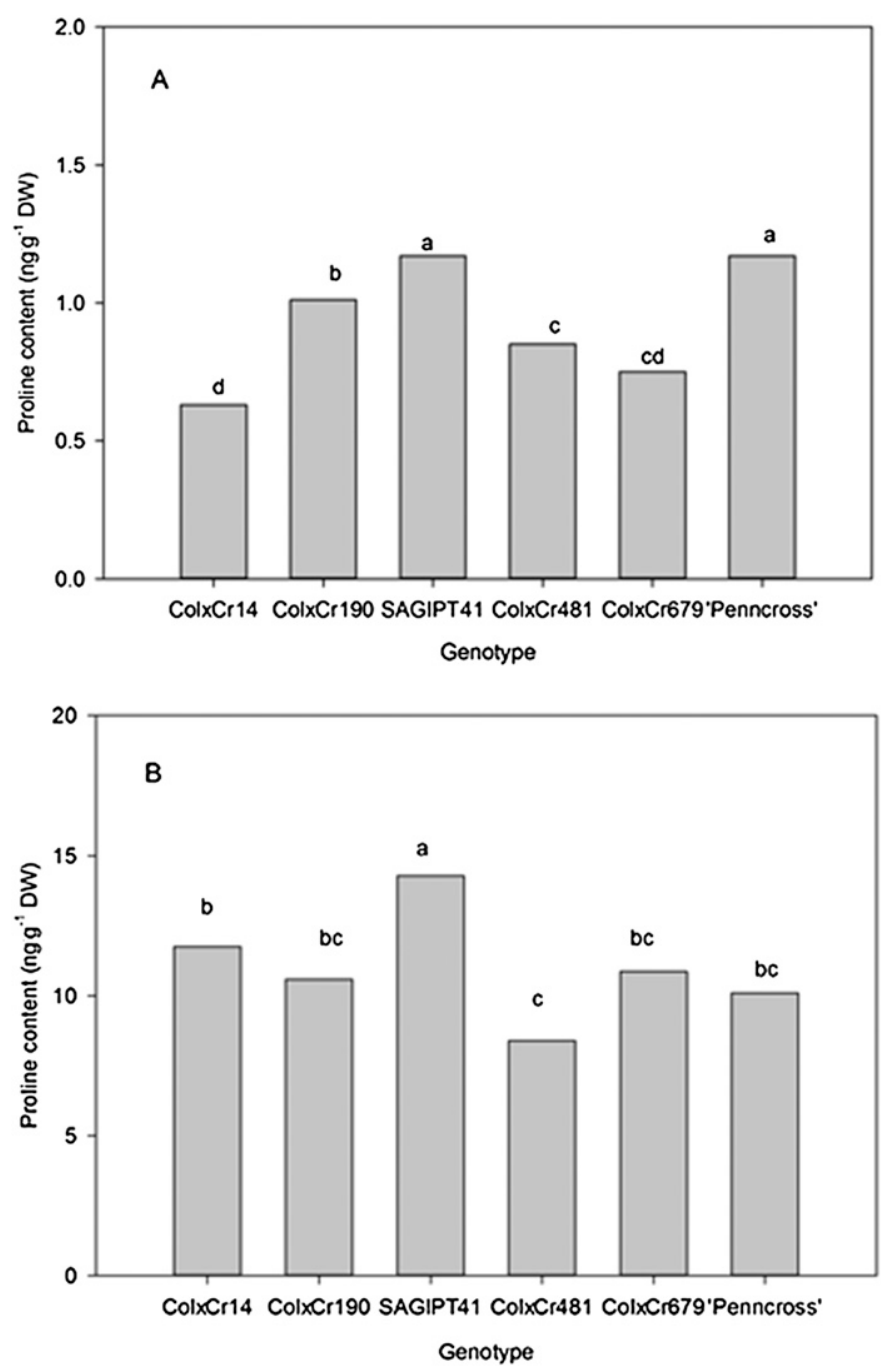

Fig. 7. Proline content of six genotypes of bentgrass under (A) well-watered conditions and (B) at $13 \mathrm{~d}$ of drought stress.

and RWC, and negatively correlated to EL. TQ, RWC, and EL were also significantly correlated with each other (Table 1).

GENOTYPIC VARIATIONS IN CONTENT OF OSMOLYTES AND CONTRIBUTION OF DIFFERENT OSMOLYTES TO OSMOTIC ADJUSTMENT. Content of osmolytes, including soluble sugars (glucose, sucrose, and fructose), proline, GB, and PA (Put, $\mathrm{Spm}$, and Spd) were measured in leaves of well-watered plants and drought-stressed plants at $13 \mathrm{~d}$ of treatment when genotypic differences in physiological parameters were most pronounced during drought periods. Different genotypes exhibited variations in the content of different osmolytes under either wellwatered or drought conditions (Figs. 5-9).

For soluble sugar content under well-watered conditions, 'Penncross' had highest glucose, sucrose, fructose, and total soluble sugar content while other genotypes were not significantly different from each other for glucose and fructose; sucrose and total soluble sugar content was lower in ColxCr679 than 'Penncross' but lower than other genotypes (Fig. 5). Under drought stress, SAGIPT41 had greatest content of glucose, sucrose, fructose, and total soluble sugar content, which was followed by ColxCr14 and ColxCr190, whereas ColxCr679 had the lowest content of those sugars. The content of glucose, sucrose, and fructose, as well as the total content of soluble sugars in ColxCr481 and 'Penncross' was significantly lower than in SAGIPT41, ColxCr14, or ColxCr190, but greater than that in ColxCr679 under drought stress (Fig. 6). Proline content for well-watered plants was ranked as SAGIPT41, ColxCr190, ColxCr481, ColxCr679, ColxCr14, 'Penncross' = SAGIPT41 > ColxCr190 $>$ ColxCr481 $=$ ColxCr679 $=$ ColxCr14 (Fig. 7A). Under drought stress, SAGIPT41 had highest proline content whereas ColxCr481 had the lowest proline content and others were intermediate (Fig. 7B).

Put content of well-watered plants was highest in ColxCr481, followed by ColxCr679, and other genotypes had equally lower levels (Fig. 8A). Spm content of well-watered plants ranked as ColxCr14, ColxCr190, ColxCr481, ColxCr679, ColxCr14 = SAGIPT41 $=\operatorname{ColxCr190>}$ ColxCr481 > 'Penncross' > ColxCr679 (Fig. 8B). For Spd content, it was ranged from high to low level as ColxCr190 > ColxCr679 > SAGIPT41 > ColxCr14 > ColxCr481 = 'Penncross' (Fig. 8C). Under well-watered conditions, total PA content followed the order from high to low as ColxCr190 > ColxCr679 $>$ SAGIPT41 > ColxCr14 $=$ ColxCr481 $>$ 'Penncross' (Fig. 8D). At $13 \mathrm{~d}$ of drought stress, Put content in ColxCr481 and ColxCr679 were significantly higher than that in 'Penncross', SAGIPT41, ColxCr190, and ColxCr14 (Fig. 9A). Spm content was highest in SAGIPT41 and lowest in ColxCr679 and Penncross, and intermediate in other genotypes (Fig. 9B). Spd content ranged from high to low as ColxCr190 $>$ ColxCr679 $>$ SAGIPT41 > ColxCr14 > ColxCr481 = 'Penncross' (Fig. 9C). Total content of PA was ranked as ColxCr190 > ColxCr679 > ColxCr481 > SAGIPT41 > ColxCr14 > 'Penncross' (Fig. 9D).

GB content was lowest in 'Penncross', intermediate in ColxCr679, and no significant differences were among other genotypes under well-watered conditions (Fig. 10A). Under drought stress, GB content was ranked in the order of ColxCr14 = SAGIPT41 $>$ ColxCr481 > ColxCr190 = 'Penncross' > ColxCr679.

Table 2 showed the contribution of each solute to OA under drought stress. Soluble sugars were the predominant osmolytes in all genotypes and lines, contributing to $43.62 \%$ to $59.32 \%$ of $\mathrm{OA}$, which was followed by GB with $4.91 \%$ to $8.46 \%$ contribution and proline with $4.06 \%$ to $4.81 \%$ contribution. Three forms of PA are minor contributors to OA, with less than $1 \%$ contribution in all genotypes.

\section{Discussion}

The maintenance of adequate leaf water status is important for proper physiological and biochemical functioning. Plants that can maintain adequate RWC for a longer period during drought stress will have the greatest likelihood of continued metabolic activities and survival. Maintaining cell membrane stability is also crucial for proper cellular functions, and EL has been widely used to estimate cell membrane stability (Blum and Ebercon, 1981; Rachmilevitch et al., 2006). The analysis of TQ, RWC, and EL demonstrated genotypic variations in drought tolerance among a transgenic line (SAGIPT41) of creeping bentgrass, a wide type of creeping bentgrass ('Penncross'), and creeping bentgrass $X$ colonial bentgrass hybrids (ColxCr14, ColxCr190, ColxCr481, and ColxCr679), with ColxCr14, SAGIPT41, and ColxCr190 exhibiting superior drought tolerance relative to ColxCr481, ColxCr679, and 'Penncross'. Previous studies with various physiological and biochemical analysis also reported improved 

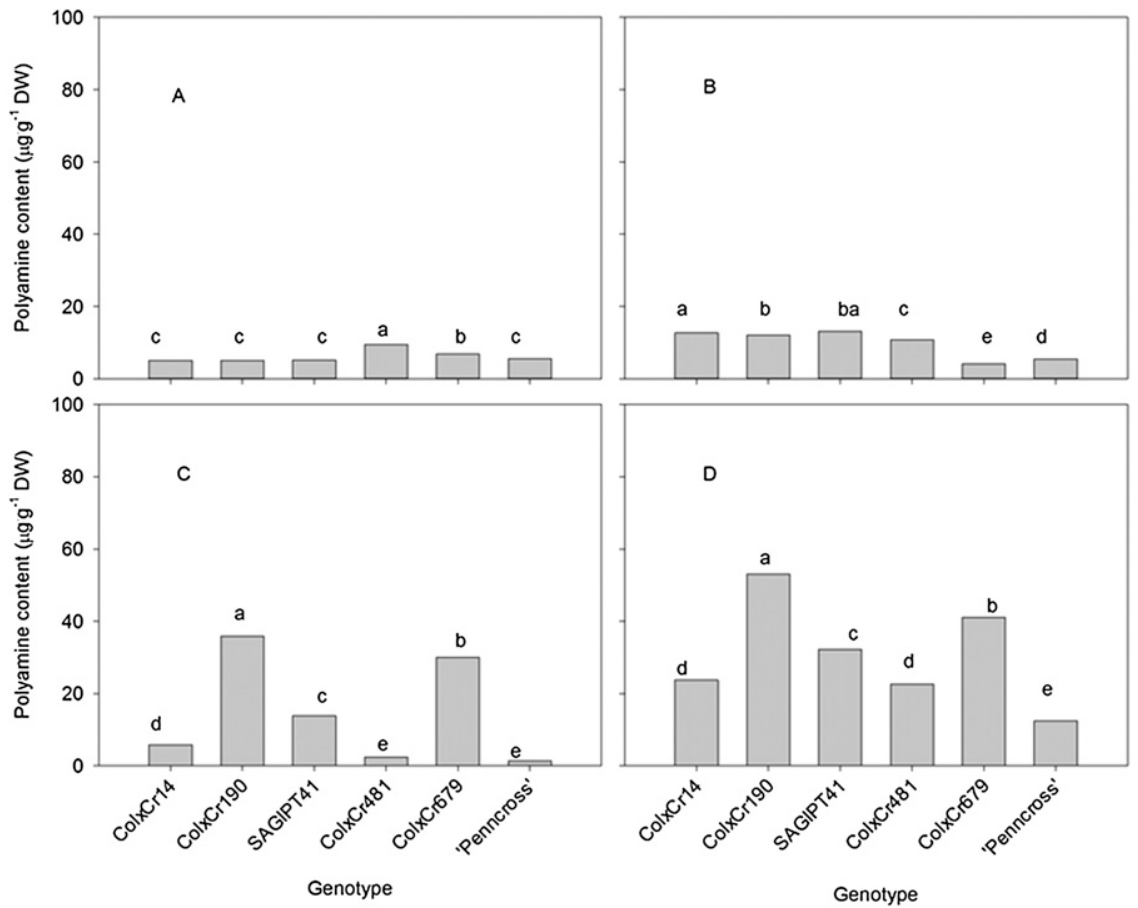

Fig. 8. Content of (A) putrescine, (B) spermidine, (C) spermidine, and (D) total polyamines for six genotypes of bentgrass under well-watered conditions. Columns marked with different letters indicate significant differences among genotypes for each parameter based on least significant difference test $(P=0.05)$.
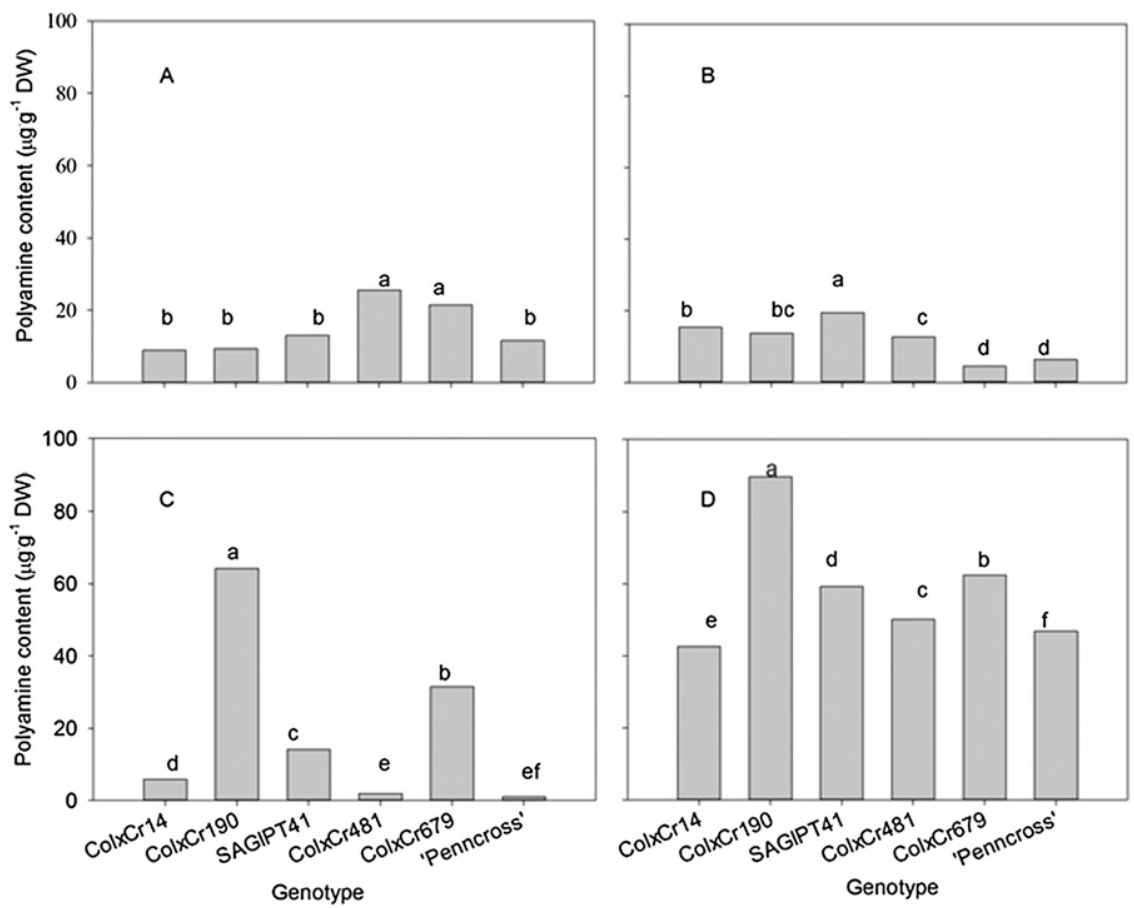

Fig. 9. Content of (A) putrescine, (B) spermidine, (C) spermidine, and (D) total polyamines for six genotypes of bentgrass at $13 \mathrm{~d}$ of drought stress. Columns marked with different letters indicate significant differences among genotypes for each parameter based on least significant difference test $(P=0.05)$.

drought tolerance of the transgenic creeping bentgrass SAGIPT41 compared with 'Penncross' (Merewitz et al., 2011, 2012).

OA has been regarded as a drought tolerance mechanism in many plants (Bohnert and Jensen, 1996; LaRosa et al., 1987), including turfgrasses (DaCosta and Huang, 2006; Qian and Fry, 1997; White et al., 1992). Increasing OA facilitates the maintenance of cell turgor under conditions of limited water availability. Our study found that drought-tolerant genotypes (ColxCr14, SAGIPT41, and ColxCr190) had higher OA than drought-sensitive ones (ColxCr481, ColxCr679, and 'Penncross'). Correlation analyses between $\mathrm{OA}$ and physiological traits demonstrated that OA was positively correlated to TQ and RWC and negatively correlated to EL. These results indicated that greater level of OA could at least partially contribute to the maintenance of better TQ and water hydration levels, and cell membrane stability under drought stress, although many physiological factors, such as photosynthesis also play essential roles in regulating plant drought tolerance (Chaves et al., 2003). OA involves the accumulation of solutes in cells that lower $\Psi_{\mathrm{s}}$, facilitating water retention or maintaining cell turgor (Boyer et al., 2008). Major types of osmolytes include soluble sugars, proline, GB, and PA (Bouchereau et al., 1999; Chaves et al., 2003; Gomes et al., 2010; Kumar et al., 1997; Martinez et al., 2004; Travert et al., 1997; Yancey et al., 1982). Soluble sugars act as osmoregulants and also play roles in stabilizing membrane structures, contributing to plant tolerance to drought stress (Bartels and Sunkar, 2005; Spollen and Nelson, 1994). Proline typically accumulates in the cytosol, and it contributes to the cytoplasmic OA in response to drought or salinity stress (Ashraf and Foolad, 2007). Many studies showed that proline was positively correlated with OA during drought stress in many other plant species (Keyvan, 2010; Quan et al., 2010; Xiong et al., 2012). GB is synthesized in response to saline or drought stress in some plant species, which regulates $\Psi_{\mathrm{s}}$ and facilitate cellular turgor maintenance (Munns, 2002). Put, Spd, and Spm are the major types of PA in plants, which play roles in OA, membrane stability, free radical scavenging and regulation of stomatal movements (Liu et al., 2008; Nayyar and Chander, 2004; Sanchez et al., 2005). In our present study, the predominant forms of osmolytes in bentgrass was soluble sugars, 

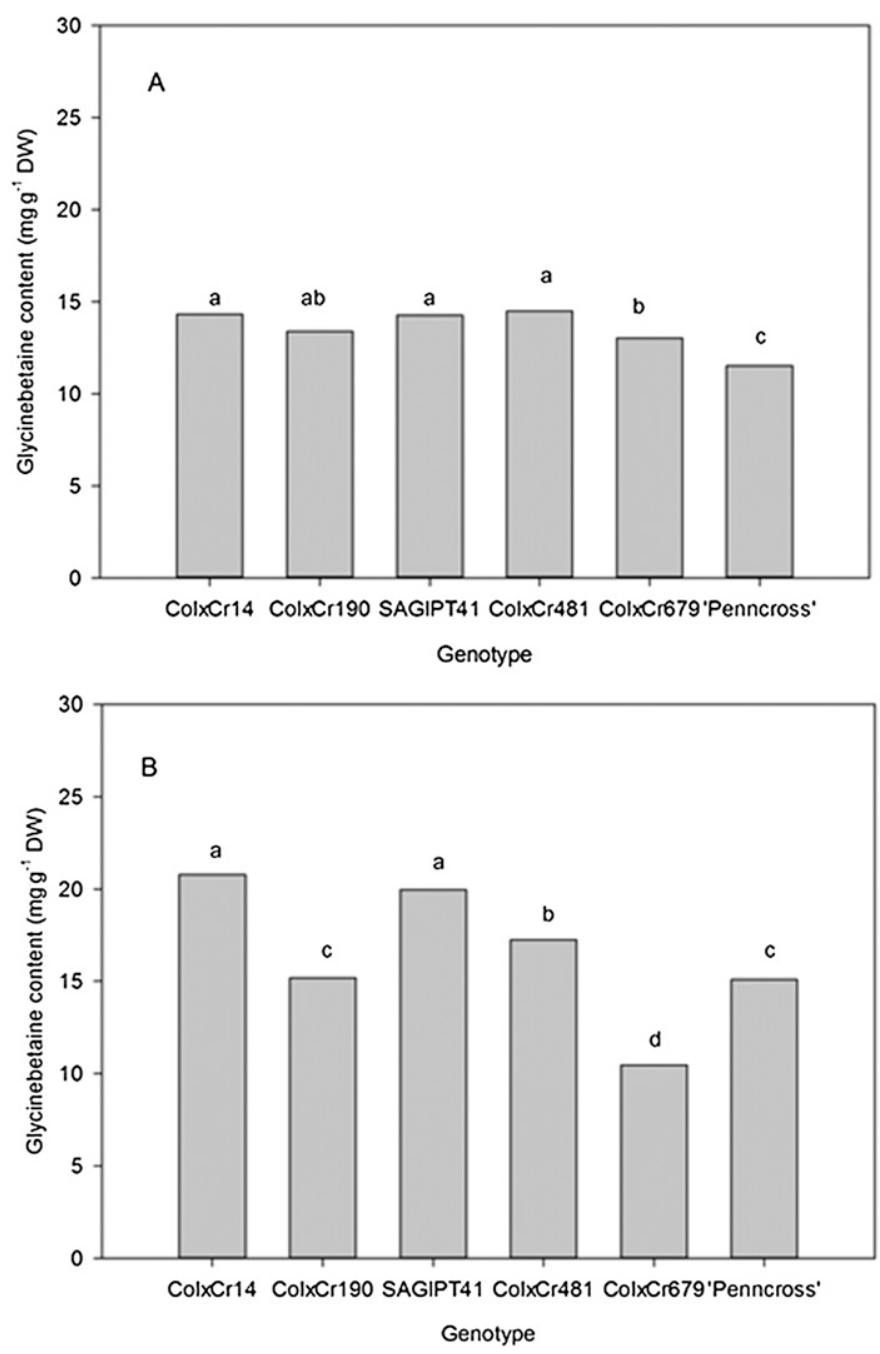

Fig. 10. Glycine betaine content of six genotypes of bentgrass under (A) wellwatered conditions and (B) at $13 \mathrm{~d}$ of drought stress.

which contributed to $43.62 \%$ to $59.32 \%$ of OA, followed by GB and proline whereas PA were relatively minor contributors to OA with less than $1 \%$ contribution.

Differential OA among genotypes could be due to the accumulation of specific or unique types of solutes in different genotypes. DaCosta and Huang (2006) reported that creeping bentgrass plants osmotically adjusted to dehydration stress by accumulating soluble carbohydrates. Jiang and Huang (2001) found kentucky bluegrass of improved drought tolerance by drought preconditioning had $21 \%$ to $44 \%$ higher soluble sugar content in leaves than nonpreconditioned, drought-sensitive plants. $\mathrm{Fu}$ et al. (2010) found that decreases in $\Psi_{\mathrm{s}}$ was accompanied by higher sucrose levels, which were the result of the increased level of sucrose phosphate synthase and sucrose synthase activity and a decline in acid invertase activity in response to drought stress. In this study, increased OA associated with better drought tolerance could be attributed to greater accumulation of soluble sugars, proline, $\mathrm{Spm}$, and GB in SAGIPT41, soluble sugars and Spd in ColxCr190, and soluble sugars and GB in ColxCr14 under drought stress, compared with the drought-sensitive genotypes, ColxCr481, ColxCr679, and 'Penncross'. The content of all solutes for plants under well-watered conditions did not exhibit consistent patterns of
Table 2. The proportion of contributions of putrescine (Put), spermidine (Spm), spermidine (Spd), proline, glycine betaine (GB), and sugars to the osmotic adjustment in bentgrass genotypes.

\begin{tabular}{lcccccc}
\hline \multicolumn{7}{c}{ Percentage of total $\Psi_{\mathrm{s}}(\%)$} \\
\hline Genotype & Put & Spm & Spd & Proline & GB & Sugar \\
\hline ColxCr14 & $0.01 \mathrm{~d}^{\mathrm{z}}$ & $0.02 \mathrm{~d}$ & $0.23 \mathrm{~d}$ & $4.70 \mathrm{c}$ & $8.18 \mathrm{~b}$ & $53.28 \mathrm{a}$ \\
ColxCr190 & $0.01 \mathrm{c}$ & $0.03 \mathrm{c}$ & $0.45 \mathrm{c}$ & $4.68 \mathrm{~b}$ & $6.60 \mathrm{~b}$ & $50.82 \mathrm{a}$ \\
SAGIPT41 & $0.01 \mathrm{~d}$ & $0.01 \mathrm{~d}$ & $0.27 \mathrm{~d}$ & $4.46 \mathrm{c}$ & $7.71 \mathrm{~b}$ & $55.13 \mathrm{a}$ \\
ColxCr481 & $0.02 \mathrm{~d}$ & $0.02 \mathrm{~d}$ & $0.29 \mathrm{~d}$ & $4.19 \mathrm{c}$ & $8.46 \mathrm{~b}$ & $58.20 \mathrm{a}$ \\
ColxCr679 & $0.02 \mathrm{c}$ & $0.02 \mathrm{c}$ & $0.26 \mathrm{c}$ & $4.81 \mathrm{~b}$ & $4.91 \mathrm{~b}$ & $43.62 \mathrm{a}$ \\
'Penncross' & $0.01 \mathrm{c}$ & $0.01 \mathrm{c}$ & $0.17 \mathrm{c}$ & $4.06 \mathrm{~b}$ & $5.96 \mathrm{~b}$ & $59.32 \mathrm{a}$
\end{tabular}

${ }^{\mathrm{z}}$ Means followed by the same letters within a line indicate no significant differences between treatments base on least significant difference test at $P=0.01$.

higher values in the drought-tolerant genotypes (SAGIPT41, ColxCr190, and ColxCr14) compared with the drought-sensitive genotypes (ColxCr481, ColxCr679, and 'Penncross'), suggesting that increased OA under drought stress was not associated with genotypic differences in solute accumulation of plants under well-watered conditions, but due to increased accumulation of solutes in response to drought stress. Although PA was found to be a minor contributor to OA in bentgrass species in this study, the significant positive correlation of Spm with OA suggested that Spm is a major form of PA associated with OA and improved drought tolerance in bentgrass species. It is worthy of noting that the sum of the contribution of osmolytes examined in this study was less than $100 \%$. That might be due to the accumulation of other solutes in the cell contributing to OA, such as polyols (e.g., mannitol), ions (e.g., potassium), and organic acids (e.g., malate) (Chaves et al., 2003; Hare et al., 1998) which were not detected in this study, and further research is needed to examine the undetected metabolites that may also contributes to $\mathrm{OA}$ in bentgrass.

In summary, physiological analysis of genotypic variations in drought tolerance demonstrated the genetic potential for developing drought-tolerant bentgrass by selecting for OA. To our knowledge, this is the first report of different types or specific osmolytes involved in regulating OA under drought stress in turfgrasses differing in drought tolerance, with greater accumulation of soluble sugars, proline, GB, and Spm in SAGIPT41, soluble sugars and Spd in ColxCr190, and soluble sugars and GB in ColxCr14, compared with the droughtsensitive genotypes, ColxCr481, ColxCr679, and 'Penncross', as shown by the physiological analysis in this study. This study also first found that soluble sugars were major contributors to $\mathrm{OA}$, followed by GB and proline, with all three forms of PA as minor contributors in bentgrass genotypes. The osmolytes highly correlated to OA and superior drought tolerance could be used as biomarkers to select for drought-tolerant germplasm of bentgrass and other cool-season turfgrass species.

\section{Literature Cited}

Arakawa, T., Y. Kita, and J.F. Carpenter. 1991. Protein-solvent interactions in pharmaceutical formulations. Pharm. Res. 8:285-291.

Ashraf, M. and M.R. Foolad. 2007. Roles of glycine betaine and proline in improving plant abiotic stress resistance. Environ. Expt. Bot. 59:206-216.

Barrs, H.D. and P.E. Weatherley. 1962. A re-examination of the relative turgidity technique for estimating water deficit in leaves. Austral. J. Biol. Sci. 15:413-428. 
Bartels, D. and R. Sunkar. 2005. Drought and salt tolerance in plants. Crit. Rev. Plant Sci. 24:23-58.

Blum, A. 1989. Osmotic adjustment and growth of barley genotypes under drought stress. Crop Sci. 29:230-233.

Blum, A. and A. Ebercon. 1981. Cell membrane stability as a measure of drought and heat tolerance in wheat. Crop Sci. 21:43-47.

Blum, A. and C.Y. Sullivan. 1986. The comparative drought resistance of landraces of sorghum and millet from dry and humid regions. Ann. Bot. 57:835-846.

Bohnert, H. and R.G. Jensen. 1996. Strategies for engineering waterstress tolerance in plants. Trends Biotechnol. 14:89-97.

Bouchereau, A., A. Aziz, F. Larher, and J. Martin-Tanguy. 1999. Polyamines and environmental challenges: Recent developments. Plant Sci. 140:103-125.

Boyer, J.S., R.A. James, R. Munns, T.A. Condon, and J.B. Passioura. 2008. Osmotic adjustment leads to anomalously low estimates of relative water content in wheat and barley. Funct. Plant Biol. 35:1172-1182.

Buysse, J. and R. Merckx. 1993. Acclimation of potato plants to polyethylene glycol-induced water deficit: II. Contents and subcellular distribution of organic solutes. J. Expt. Bot. 49:13611370.

Chaves, M.M., J.P. Maroco, and J.S. Pereira. 2003. Understanding plant responses to drought - from genes to the whole plant. Funct. Plant Biol. 30:239-264.

DaCosta, M. and B. Huang. 2006. Changes in carbon partitioning and accumulation patterns during drought and recovery for colonial bentgrass, creeping bentgrass, and velvet bentgrass. J. Amer. Soc. Hort. Sci. 131:484-490.

Fu, J., B. Huang, and J. Fry. 2010. Osmotic potential, sucrose level, and activity of sucrose metabolic enzymes in tall fescue in response to deficit irrigation. J. Amer. Soc. Hort. Sci. 135:506-510.

Gomes, F.P., M.A. Oliva, M.S. Mielke, A.A.F. Almeida, and L.A. Aquino. 2010. Osmotic adjustment, proline accumulation and cell membrane stability in leaves of Cocos nucifera submitted to drought stress. Sci. Hort. 126:379-384.

Hare, P.D., W.A. Cress, and J. Van Staden. 1998. Dissecting the roles of osmolyte accumulation during stress. Plant Cell Environ. 21:535553.

Hoagland, D.R. and D.I. Arnon. 1950. The water-culture method for growing plans without soil. California Agr. Expt. Sta. Circ. $347: 1-32$.

Hoekstra, F.A., E.A. Golovina, and J. Buitink. 2001. Mechanisms of plant desiccation tolerance. Trends Plant Sci. 6:431-438.

Jiang, Y. and B. Huang. 2001. Osmotic adjustment and root growth associated with drought preconditioning-enhanced heat tolerance in kentucky bluegrass. Crop Sci. 41:1168-1173.

Keyvan, S. 2010. The effects of drought stress on yield, relative water content, proline, soluble carbohydrates and chlorophyll of bread wheat cultivars. J. Animal Plant Sci. 8:1051-1060.

Kumar, A., M. Taylor, T. Altabella, and A.F. Tiburcio. 1997. Recent advances in polyamine research. Trends Plant Sci. 2:124-130.

LaRosa, C.P., P.M. Hasegawa, D. Rhodes, J.M. Clithero, A.A. Watad, and R.A. Bressan. 1987. Abscisic acid stimulated osmotic adjustment and its involvement in adaptation to tobacco cells to $\mathrm{NaCl}$. Plant Physiol. 85:174-181.

Liu, J., X.J. Ji, and Y.L. Liu. 2002. High performance liquid chromatography method for measuring polyamine content in plant tissue. Plant Physiol. Commun. 38:596-598.

Liu, J.H., H. Inoue, and T. Moriguchi. 2008. Salt stress-mediated changes in free polyamine titers and expression of genes responsible for polyamine biosynthesis of apple in vitro shoots. Environ. Expt. Bot. 62:28-35.

Magné, C. and F. Larher. 1992. High sugar content of extracts interferes with colorimetric determination of amino acids and free proline. Anal. Biochem. 200:115-118.

Martinez, J., S. Lutts, A. Schanck, M. Bajji, and J.M. Kinet. 2004. Is osmotic adjustment required for water stress resistance in the Mediterranean shrub Atriplex halimus L. J. Plant Physiol. 161:1041-1051.

Merewitz, E.B., F.C. Belanger, S.E. Warnke, and B. Huang. 2012. Identification of quantitative trait loci linked to drought tolerance in a colonial $\times$ creeping bentgrass hybrid population. Crop Sci. 52:1891-1901.

Merewitz, E.B., T. Gianfagna, and B. Huang. 2011. Photosynthesis, water use, and root viability under water stress as affected by expression of SAG12-ipt controlling cytokinin synthesis in Agrostis stolonifera. J. Expt. Bot. 62:383-395.

Munns, R. 2002. Comparative physiology of salt and water stress. Plant Cell Environ. 25:239-250.

Nayyar, H. and S. Chander. 2004. Protective effects of polyamines against oxidative stress induced by water and cold stress in chickpea. J. Agron. Crop Sci. 190:355-365.

Qian, Y. and J.D. Fry. 1997. Water relations and drought tolerance of four turfgrasses. J. Amer. Soc. Hort. Sci. 122:129-133.

Qian, Y., J.D. Fry, and W.S. Upham. 1997. Rooting and drought avoidance of warm-season turfgrasses and tall fescue in Kansas. Crop Sci. 37:905-910.

Quan, R., S. Hu, Z. Zhang, H. Zhang, and R. Huang. 2010. Overexpression of an ERF transcription factor TSRF1 improves rice drought tolerance. Plant Biotechnol. J. 8:476-488.

Rachmilevitch, S., M. DaCosta, and B. Huang. 2006. Physiological and biochemical indicators for stress tolerance, p. 321-356. In: B. Huang (ed.). Plant-environment interactions. 3rd ed. CRC Press, Boca Raton, FL.

Rhodes, D. and A.D. Hanson. 1993. Quaternary ammonium and tertiary sulfonium compounds in higher plants. Annu. Rev. Plant Physiol. 44:357-384.

Sanchez, D.H., J.C. Cuevas, M.A. Chiesa, and O.A. Ruiz. 2005. Free spermidine and spermine contents in Lotus glaber under long-term salt stress. Plant Sci. 168:541-546.

Spollen, W.G. and C.J. Nelson. 1994. Response of fructan to water deficit in growing leaves of tall fescue. Plant Physiol. 106:329-336.

Travert, S., L. Valerio, I. Fouraste, A.M. Boudet, and C. Teulieres. 1997. Enrichment in specific soluble sugars of two eucalyptus cellsuspension cultures by various treatments enhances their frost tolerance via a noncolligative mechanism. Plant Physiol. 114:1433-1442.

Turgeon, A.J. 2008. Turfgrass management. 8th ed. Pearson Prentice Hall, Upper Saddle River, NJ.

Wang, X., Y. Wu, and L.M. Wang. 2010. Determination of betaine in fruits of Lycium barbarum L. by solid phase extraction and highperformance liquid chromatography. Food Sci. 8:199-201.

White, R.H., M.C. Engelke, S.J. Morton, and B.A. Ruemmele. 1992. Competitive turgor maintenance in tall fescue. Crop Sci. 32:251-256.

Xiong, J., L. Zhang, G. Fu, Y. Yang, C. Zhu, and L. Tao. 2012. Drought-induced proline accumulation is uninvolved with increased nitric oxide, which alleviates drought stress by decreasing transpiration in rice. J. Plant Res. 125:155-164.

Yancey, P.H., M.E. Clark, S.C. Hand, R.D. Bowlus, and G.N. Somero. 1982. Living with water stress: Evolution of osmolyte systems. Science 217:1214-1222. 